Physical Chemistry

Improvements on a pulsed slit jet-cavity ring down experiment

\author{
Carine Manca, Martin Suter, Andreas Schneider, Martin Quack
}

Physical Chemistry, ETH Zurich, 8093 Zurich, Switzerland

Cavity ring down spectroscopy with a continuous wave laser is an interesting technique since it allows direct measurements of absorbance, very high resolution and extreme sensitivity [1-4]. When it is combined with pulsed supersonic slit jet expansion, it reduces Doppler broadening, and pressure broadening and congestions arising from vibrational and rotational hot bands are largely removed. The assignment of highly resolved spectra is simplified. The combination of these two techniques has successfully been developed in our group to measure combination bands of nitrous oxide, chloroform, and methane [1,2]. The trigger system uses a simple passive scheme, where the cavity length is periodically changed so that the cavity length matches the laser wavelength [1-3]. Our techniques have also been recently successfully adopted by other groups [5-6].

Here we present new improvements on the apparatus in our laboratory. Both the trigger system and the jet expansion have been optimized to allow better quantitative measurements. Also the development of a new data acquisition is in progress: it controls all the instruments, allows a regulation of the jet expansion in the cavity and increases the signal to noise ratio. We shall report on the experimental aspects of these developments and on results of ongoing experiments.

[1] M. Hippler and M. Quack, J. Chem. Phys. 2002, 116, 6045.

[2] Y. He, M. Hippler, and M. Quack, Chem. Phys. Lett. 1998, 289, 527.

[3] M. Hippler and M. Quack, Chem. Phys. Lett. 1999, 314, 273.

[4] D. Romanini, A. A. Kachanov, and F. Stoeckel, Chem. Phys. Lett. 1997, 270, 538; B. A. Paldus et al., J. Appl. Phys. 1998, 83, 3991.

[5] P. Birza, T. Motylewski, D. Khoroshev, A. Chirokolava, H. Linnartz, J. P. Maier, Chem. Phys. 2002, 283, 119

[6] S. C. Xu, J. J. Kay, D. S. Perry, J. Mol. Spec. 2004, 225, 162.

\section{Polymer Science}

Block Copolymer Self-Assembly Involving Competing Length Scales

\section{Gerrit ten Brinke}

University of Groningen, Zernike Institute for Advanced Materials, Nijenborgh 4, 9747 AG Groningen, The Netherlands

Self-assembly in block copolymer-based systems resulting in hierarchically ordered structures characterized by two different length scales have been realized in several systems during the last decade. These systems usually involve three chemically different moieties and in combination with a polymer chain architecture that involves two different length scales, e.g. a diblock copolymer consisting of a linear homopolymer block and a comb copolymer block, the formation of such structures is quite obvious. When aiming at structure formation involving a genuine competition between different length scales we need to restrict ourselves to binary block copolymers, i.e. block copolymers involving only two chemically different moieties, with a molecular architecture characterized by two length scales. The simplest example consists of linear $\mathrm{A}_{m}-b-(\mathrm{A}-b-\mathrm{B})_{n}-\mathrm{B}_{m}$ symmetric multiblock copolymers. Here A and B represent chemically different "short" flexible chains of equal length. The parameters $m$ and $n$ denote the length of the end blocks and the number of symmetric A- $b$-B diblocks of the middle multiblock respectively.

Interesting new phenomena that occur within the $(m, n)$-parameter space include: a homogeneous melt characterized by a small angle X-ray scattering pattern with two scattering peaks, complex self-assembled structures characterized by two different length scales, several self-assembled structures with the cubic symmetry, i.e. face-centered cubic, body-centered cubic, simple cubic and "single" gyroid, and a variety of order-order transitions between different ordered structures involving a large and discontinuous change in the periodicity length scale. These results demonstrate that this new class of materials should have great potential in the area of responsive nanomaterials, e.g. temperature switching functional properties.
Physical Chemistry

\section{Global Analysis of $\mathrm{CH}_{4}$ Lines in the $0-3200 \mathrm{~cm}^{-1}$ Region}

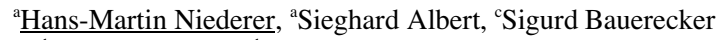

${ }^{\mathrm{b}}$ Vincent Boudon, ${ }^{\mathrm{b}}$ Jean-Paul Champion, ${ }^{\mathrm{a}}$ Martin Quack

${ }^{a}$ ETH Zürich, Wolfgang-Pauli-Strasse 10, CH-8093 Zürich, Switzerland b Institut Carnot de Bourgogne, 9 Av. A. Savary, F-21078 Dijon, France ${ }^{\mathrm{C}}$ TU Braunschweig, Hans-Sommer-Strasse 10, D-38106 Braunschweig, Germany

We have carried out new measurements of infrared spectra of the spherical top molecules ${ }^{12} \mathrm{CH}_{4},{ }^{13} \mathrm{CH}_{4}$ and ${ }^{12} \mathrm{CD}_{4}$ at low temperature $(80 \mathrm{~K})$ and at very high resolution $\left(0.0027 \mathrm{~cm}^{-1}\right.$ for spectra in the pentad region around 2900 $\mathrm{cm}^{-1}$ ) using the Zürich prototype Bruker 125 spectrometer (ZP 2001) [1] combined with a low temperature cooling cell [2]. Due to low temperature, the Doppler full width at half maximum was reduced by a factor of 1.91 to $0.0045 \mathrm{~cm}^{-1}$ in the pentad region. We report the global analysis of ${ }^{13} \mathrm{CH}_{4}$ transitions up to $3200 \mathrm{~cm}^{-1}$ and compare to previous work [3]. In the present work line positions are reproduced with an rms deviation of $0.0009 \mathrm{~cm}^{-1}$. The complex interacting system is analyzed using an effective Hamiltonian theory described in [4]. We also discuss an introduction into the tensorial formalism [5].

[1] S. Albert, K. K. Albert and M. Quack, Trends in Optics and Photonics, 2003, 84, 177-180.

[2] S. Bauerecker, M. Taraschewski, C. Weitkamp and H. K. Cammenga, Rev. Sci. Instruments, 2001, 72, 3946-3955.

[3] J. M. Jouvard, B. Lavorel, J. P. Champion and L. R. Brown, J. Mol. Spec., 1991, 150, 201-217.

[4] J. P. Champion, M. Loëte and G. Pierre in Spectroscopy of the Earth's Atmosphere and Interstellar Medium, edited by K. N. Rao and A. Weber, 1992, Academic Press, San Diego.

[5] A. Frank and P. van Isacker, Algebraic Methods in Molecular and Nuclear Structure Physics, 1994, John Wiley \& Sons, New York.

Polymer Science

Mesoscale dynamics in nanostructured polymeric fluids

\section{$\underline{\text { Robert Magerle }}$}

Technische Universität Chemnitz, Reichenhainer Str. 70, 09107 Chemnitz, Germany

With tapping mode scanning force microscopy we observe the microdomain dynamics at the surface of thin films of block copolymer melts and concentrated solutions. A high image acquisition rate and the correction of image distortions with non-linear registration techniques enable a new view onto fundamental processes of structure formation in melts and concentrated solutions of block copolymers and multi-component polymeric fluids. One example is the structural phase transition from cylinders to a hexagonal perforated lamella in a thin film of a concentrated solution of polystyreneblock-polybutadiene-block-polystyrene in chloroform [1]. From the kinetics of the phase transition we could estimate the interfacial tension between the two differently ordered fluids. Other experiments show correlations between the dynamics of neighboring defects [2], annealing of defects via formation of transient phases [3], shape fluctuations of cylinders [3,4] and lamellae [4], as well as the dynamics of individual perforations in a hexagonally perforated lamellae [4].

[1] A. Knoll, A. Horvat, K. S. Lyakhova, G. Krausch, G. J. A. Sevink, A. V. Zvelindovsky, and R. Magerle, Nature Materials 2004, 3, 886.

[2] L. Tsarkova, A. Knoll, and R. Magerle, Nano Letters 2006, 6, 1574.

[3] L. Tsarkova, A. Horvat, G. Krausch, A. V. Zvelindovsky, G. J. A. Sevink, and R. Magerle, Langmuir 2006, 22, 8089.

[4] M. Kreis, Diploma Thesis, Universität Bayreuth, 2005. 
Physical Chemistry

Improvements on a pulsed slit jet-cavity ring down experiment

\author{
Carine Manca, Martin Suter, Andreas Schneider, Martin Quack
}

Physical Chemistry, ETH Zurich, 8093 Zurich, Switzerland

Cavity ring down spectroscopy with a continuous wave laser is an interesting technique since it allows direct measurements of absorbance, very high resolution and extreme sensitivity [1-4]. When it is combined with pulsed supersonic slit jet expansion, it reduces Doppler broadening, and pressure broadening and congestions arising from vibrational and rotational hot bands are largely removed. The assignment of highly resolved spectra is simplified. The combination of these two techniques has successfully been developed in our group to measure combination bands of nitrous oxide, chloroform, and methane [1,2]. The trigger system uses a simple passive scheme, where the cavity length is periodically changed so that the cavity length matches the laser wavelength [1-3]. Our techniques have also been recently successfully adopted by other groups [5-6].

Here we present new improvements on the apparatus in our laboratory. Both the trigger system and the jet expansion have been optimized to allow better quantitative measurements. Also the development of a new data acquisition is in progress: it controls all the instruments, allows a regulation of the jet expansion in the cavity and increases the signal to noise ratio. We shall report on the experimental aspects of these developments and on results of ongoing experiments.

[1] M. Hippler and M. Quack, J. Chem. Phys. 2002, 116, 6045.

[2] Y. He, M. Hippler, and M. Quack, Chem. Phys. Lett. 1998, 289, 527.

[3] M. Hippler and M. Quack, Chem. Phys. Lett. 1999, 314, 273.

[4] D. Romanini, A. A. Kachanov, and F. Stoeckel, Chem. Phys. Lett. 1997, 270, 538; B. A. Paldus et al., J. Appl. Phys. 1998, 83, 3991.

[5] P. Birza, T. Motylewski, D. Khoroshev, A. Chirokolava, H. Linnartz, J. P. Maier, Chem. Phys. 2002, 283, 119

[6] S. C. Xu, J. J. Kay, D. S. Perry, J. Mol. Spec. 2004, 225, 162.

\section{Polymer Science}

Block Copolymer Self-Assembly Involving Competing Length Scales

\section{Gerrit ten Brinke}

University of Groningen, Zernike Institute for Advanced Materials, Nijenborgh 4, 9747 AG Groningen, The Netherlands

Self-assembly in block copolymer-based systems resulting in hierarchically ordered structures characterized by two different length scales have been realized in several systems during the last decade. These systems usually involve three chemically different moieties and in combination with a polymer chain architecture that involves two different length scales, e.g. a diblock copolymer consisting of a linear homopolymer block and a comb copolymer block, the formation of such structures is quite obvious. When aiming at structure formation involving a genuine competition between different length scales we need to restrict ourselves to binary block copolymers, i.e. block copolymers involving only two chemically different moieties, with a molecular architecture characterized by two length scales. The simplest example consists of linear $\mathrm{A}_{m}-b-(\mathrm{A}-b-\mathrm{B})_{n}-\mathrm{B}_{m}$ symmetric multiblock copolymers. Here A and B represent chemically different "short" flexible chains of equal length. The parameters $m$ and $n$ denote the length of the end blocks and the number of symmetric A- $b$-B diblocks of the middle multiblock respectively.

Interesting new phenomena that occur within the $(m, n)$-parameter space include: a homogeneous melt characterized by a small angle X-ray scattering pattern with two scattering peaks, complex self-assembled structures characterized by two different length scales, several self-assembled structures with the cubic symmetry, i.e. face-centered cubic, body-centered cubic, simple cubic and "single" gyroid, and a variety of order-order transitions between different ordered structures involving a large and discontinuous change in the periodicity length scale. These results demonstrate that this new class of materials should have great potential in the area of responsive nanomaterials, e.g. temperature switching functional properties.
Physical Chemistry

\section{Global Analysis of $\mathrm{CH}_{4}$ Lines in the $0-3200 \mathrm{~cm}^{-1}$ Region}

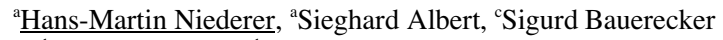

${ }^{\mathrm{b}}$ Vincent Boudon, ${ }^{\mathrm{b}}$ Jean-Paul Champion, ${ }^{\mathrm{a}}$ Martin Quack

${ }^{a}$ ETH Zürich, Wolfgang-Pauli-Strasse 10, CH-8093 Zürich, Switzerland b Institut Carnot de Bourgogne, 9 Av. A. Savary, F-21078 Dijon, France ${ }^{\mathrm{C}}$ TU Braunschweig, Hans-Sommer-Strasse 10, D-38106 Braunschweig, Germany

We have carried out new measurements of infrared spectra of the spherical top molecules ${ }^{12} \mathrm{CH}_{4},{ }^{13} \mathrm{CH}_{4}$ and ${ }^{12} \mathrm{CD}_{4}$ at low temperature $(80 \mathrm{~K})$ and at very high resolution $\left(0.0027 \mathrm{~cm}^{-1}\right.$ for spectra in the pentad region around 2900 $\mathrm{cm}^{-1}$ ) using the Zürich prototype Bruker 125 spectrometer (ZP 2001) [1] combined with a low temperature cooling cell [2]. Due to low temperature, the Doppler full width at half maximum was reduced by a factor of 1.91 to $0.0045 \mathrm{~cm}^{-1}$ in the pentad region. We report the global analysis of ${ }^{13} \mathrm{CH}_{4}$ transitions up to $3200 \mathrm{~cm}^{-1}$ and compare to previous work [3]. In the present work line positions are reproduced with an rms deviation of $0.0009 \mathrm{~cm}^{-1}$. The complex interacting system is analyzed using an effective Hamiltonian theory described in [4]. We also discuss an introduction into the tensorial formalism [5].

[1] S. Albert, K. K. Albert and M. Quack, Trends in Optics and Photonics, 2003, 84, 177-180.

[2] S. Bauerecker, M. Taraschewski, C. Weitkamp and H. K. Cammenga, Rev. Sci. Instruments, 2001, 72, 3946-3955.

[3] J. M. Jouvard, B. Lavorel, J. P. Champion and L. R. Brown, J. Mol. Spec., 1991, 150, 201-217.

[4] J. P. Champion, M. Loëte and G. Pierre in Spectroscopy of the Earth's Atmosphere and Interstellar Medium, edited by K. N. Rao and A. Weber, 1992, Academic Press, San Diego.

[5] A. Frank and P. van Isacker, Algebraic Methods in Molecular and Nuclear Structure Physics, 1994, John Wiley \& Sons, New York.

Polymer Science

Mesoscale dynamics in nanostructured polymeric fluids

\section{$\underline{\text { Robert Magerle }}$}

Technische Universität Chemnitz, Reichenhainer Str. 70, 09107 Chemnitz, Germany

With tapping mode scanning force microscopy we observe the microdomain dynamics at the surface of thin films of block copolymer melts and concentrated solutions. A high image acquisition rate and the correction of image distortions with non-linear registration techniques enable a new view onto fundamental processes of structure formation in melts and concentrated solutions of block copolymers and multi-component polymeric fluids. One example is the structural phase transition from cylinders to a hexagonal perforated lamella in a thin film of a concentrated solution of polystyreneblock-polybutadiene-block-polystyrene in chloroform [1]. From the kinetics of the phase transition we could estimate the interfacial tension between the two differently ordered fluids. Other experiments show correlations between the dynamics of neighboring defects [2], annealing of defects via formation of transient phases [3], shape fluctuations of cylinders [3,4] and lamellae [4], as well as the dynamics of individual perforations in a hexagonally perforated lamellae [4].

[1] A. Knoll, A. Horvat, K. S. Lyakhova, G. Krausch, G. J. A. Sevink, A. V. Zvelindovsky, and R. Magerle, Nature Materials 2004, 3, 886.

[2] L. Tsarkova, A. Knoll, and R. Magerle, Nano Letters 2006, 6, 1574.

[3] L. Tsarkova, A. Horvat, G. Krausch, A. V. Zvelindovsky, G. J. A. Sevink, and R. Magerle, Langmuir 2006, 22, 8089.

[4] M. Kreis, Diploma Thesis, Universität Bayreuth, 2005. 
Polymer Science

Formation of giant amphiphiles by post-functionalization of hydrophilic polymers.

\section{B. Le Droumaguet, ${ }^{\text {a }}$ G. Mantovani, ${ }^{\text {b }}$ D. M. Haddleton, ${ }^{\text {b K. Kelo- }}$} nia $^{*{ }^{*}}$

${ }^{a}$ University of Geneva, Sciences II, CH-1211 Geneva 4, Switzerland. ${ }^{b}$ University of Warwick, CV4 7AL, Coventry, UK.

A novel, generic synthetic approach leading to the first tri-block proteinpolymer amphiphiles is introduced [1]. More specifically, we designed and synthesized a multifunctional hydrophilic poly-1-alkyne polymer (I) which was initially coupled to protein molecules to form hydrophilic multifunctional tri-block biohybrids. Following our post-functionalization approach, a number of hydrophobic groups was subsequently introduced utilizing a multiple [3+2]-Huisgen cycloaddition "clicking" step.

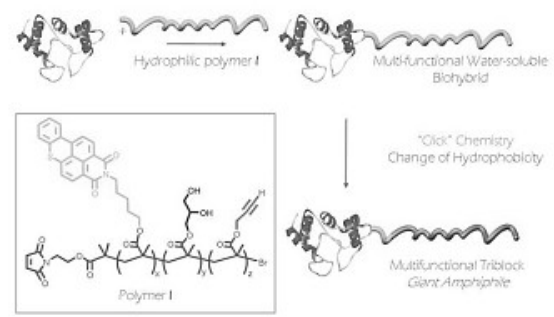

Herein we report on the family of Giant Amphiphiles resulting from the coupling of a variety of azide appended derivatives $(\mathrm{R}=\mathrm{n}$-decane, benzyl, pentafluorobenzyl, thymine) to BSA multifunctional biohybrids. A comparative study of the self-assembling behaviour of these macromolecules revealed interesting differences in the aggregation, depending on the nature of the hydrophobic derivative clicked on the polymer backbone.

[1] B. Le Droumaguet, G. Mantovani, D.M. Haddleton, K. Velonia, J. Mater. Chem. 2007, in press DOI: 10.1039/b618079e.

\section{Polymer Science}

The thermal polymerization of amino acids revisited; Synthesis and structural characterization of hyperbranched polymers from $L$-lysine

\section{Markus Scholl, ${ }^{\mathrm{a}}$ Tuan Q. Nguyen, ${ }^{\mathrm{a}}$ Bernd Bruchmann, ${ }^{\mathrm{b}}$ Harm-Anton Klok ${ }^{\mathrm{a}^{*}}$}

${ }^{a}$ École Polytechnique Fédérale de Lausanne, Institut des Matériaux, Laboratoire des Polymères, Bâtiment MXD, Station 12, CH-1015 Lausanne, Switzerland.

${ }^{b}$ BASF Aktiengesellschaft, Polymer Research, D-67056 Ludwigshafen, Germany.

The thermal polymerization of $L$-lysine hydrochloride was reinvestigated with regards to its feasibility for the synthesis of hyperbranched polylysine. ${ }^{1}$ Polymerization of the $L$-lysine* $\mathrm{HCl}$ in the presence of 1 equivalent $\mathrm{KOH}$ at $150{ }^{\circ} \mathrm{C}$ for 48 hours afforded a polymer with $\mathrm{M}_{\mathrm{N}}=4700 \mathrm{~g} / \mathrm{mol}$ and $\mathrm{M}_{\mathrm{W}} / \mathrm{M}_{\mathrm{N}}$ $=2.7$. The rate of polymerization and the polymer molecular weight could be significantly increased by adding $3 \mathrm{~mol} \% \mathrm{Zr}\left(\mathrm{O}^{\mathrm{n}} \mathrm{Bu}\right)_{4}$ yielding in polymers with $\mathrm{M}_{\mathrm{N}}=6000 \mathrm{~g} / \mathrm{mol}$ and $\mathrm{M}_{\mathrm{W}} / \mathrm{M}_{\mathrm{N}}=3.2$ after 48 hours at $150{ }^{\circ} \mathrm{C}$. ${ }^{1}$ H-NMR spectroscopy provided unequivocal proof for the hyperbranched structure of the polylysine and allowed quantification of the relative amounts of the different structural units. The degree of branching (DB) and average number of branches (ANB) for the hyperbranched polylysines varied between $0.35-0.45$, respectively $0.15-0.25$. Numerical simulations of the time dependence of the mole fractions of the different structural units suggested that the rate constant for polymerization via the $\varepsilon-\mathrm{NH}_{2}$ group was 2-4 times larger than the rate constant for polymerization via the $\alpha-\mathrm{NH}_{2}$ group. This is in good agreement with NMR results, which indicated that the mole fraction of $\varepsilon$-linked linear units is approximately 2.5 times larger than the fraction of $\alpha$-linked linear units.

[1] M. Scholl, T. Q. Nguyen, B. Bruchmann, H.-A. Klok, submitted for publication.
Polymer Science

Effect of filler dispersion on the mechanical properties in polystyrene/clay nanocomposite films.

Riccardo Ruggerone *. Christopher J.G. Plummer *, Elodie Bourgeat-Lami **, Jan-Anders E. Månson*

*Laboratoire de Technologie des Composites et Polymères (LTC), STI-IMX, Ecole Polytechnique Fédérale de Lausanne (EPFL) CH-1015 Lausanne, Switzerland

** CPE, CNRS, Laboratoire de Chimie \& Procédés de Polymérisation, Bat F 308,43 Bd 11 Novembre 1918,BP 2177, Villeurbanne, F-69616 France

This investigation concerns the mechanical properties of relatively thin nanocomposite films (about $300 \mu \mathrm{m}$ ) derived from novel styrenic latexes and containing up to $20 \mathrm{wt} \%$ laponite clay. Emulsion polymerization permits good control over the dispersion of the laponite, taking advantage of its tendency to swell in aqueous media [1]. The films were obtained by drying followed by compression molding, and were subjected to dynamic mechanical analysis and fracture tests. The results indicated a significantly higher storage modulus in the presence of the laponite both in the glassy state and in the rubbery state. However, whereas the modulus in the rubbery state increased monotonically with the nanofiller content, below and in the vicinity of the glass transition temperature, it is argued to depend critically on the degree of dispersion of the laponite particles, as determined by TEM observation of thin sections from the films. The results are discussed in terms of estimates of the extent of the polymer-laponite interphase and the classical models for stiffness reinforcement in particle filled composites.

[1] N.N. Herrera, J.M. Letoffe, J.L. Putaux, L. David, E. BourgeatLami, Langmuir. 2004, 20, 1564.

Polymer Science

404

RAFT homo- and co-polymerization of acryloyl-morpholine, piperidine, azocane and their self-assembled structures

$\underline{\text { Yun Suk Jo }}^{\dagger}$, André van der Vlies ${ }^{\dagger}$, Jay Gantz ${ }^{\dagger}$, Sasa Antonijevic ${ }^{\ddagger}$, Diana Velluto $^{\dagger}$, Jeffrey A. Hubbell ${ }^{\dagger, \ldots, *}$

Institute of Bioengineering (IBI) ${ }^{\dagger}$ and Institute of Chemical Sciences and Engineering (ISIC) $)^{\ddagger}$ École Polytechnique Fédérale de Lausanne (EPFL), 1015 Lausanne, Switzerland (E-mail: jeffrey.hubbell@epfl.ch)

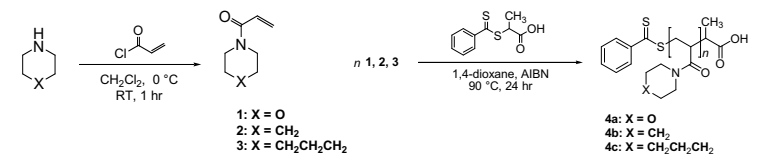

Polymeric aggregates are widely studied as drug delivery systems (DDS) in biomedical research [1][2]. We present new polymeric amphiphiles derived from $\mathrm{N}$-acryloyl derivatives of the cyclic secondary amines morpholine, piperidine, and azocane formed by reversible addition-fragmentation transfer (RAFT) polymerization [3]. Both homopolymerization and block copolymerization were carried out. In all cases, the degree of polymerization was well controlled and polymers were yielded in a monomodal distribution. The macroamphiphile aggregates in water were reproducibly well-formed by dialysis with a size range between 10 and $70 \mathrm{~nm}$ as characterized by dynamic light scattering (DLS), and the morphology of the aggregates was examined by cryogenic transmission electron microscopy (cryoTEM).

[1] K. Kataoka, A. Harada, Y. Nagasaki, Adv. Drug Delivery Rev. 2001, 47, 113.

[2] J.A. Hubbell, Science 2003, 300, 595.

[3] J. Chiefari, Y.K. Chong, F. Ercole, J. Krstina, J. Jeffery, T.P.T. Le, R.T.A. Mayadunne, G.F. Meijs, C.L. Moad, G. Moad, E. Rizzardo, S.H. Thang, Macromolecules 1998, 31, 5559. 
PEG-b-PPS diblock copolymers micelles as vehicles of hydrophobic drugs

\section{Diana Velluto $^{a}$, Davide Demurtas ${ }^{\mathrm{b}}$, Jeffrey A. Hubbell ${ }^{\mathrm{a}}$}

${ }^{a}$ Laboratory of Regenerative Medecine and Pharmacobiology, Bioingineeing Institute (LMRP), École Polytechnique Fédérale de Lausanne,

${ }^{\mathrm{b}}$ Centre of Electron Microscopy and Laboratory of Ultrastructural Analysis $(L A U)$, Faculty of Biology and Medicine, University of Lausanne

Micelles formed from amphiphilic block copolymers have been explored in recent years as carriers for hydrophobic drugs ${ }^{1}$. In an aqueous environment, the hydrophobic blocks form the core of the micelle, which can host lipophilic drugs, while the hydrophilic blocks form the corona or outer shell stabilizing interface between the hydrophobic core and the external medium In the present work two highly hydrophobic immunosuppressive drugs, $\mathrm{Cy}$ closporin A (CsA) and Everolimus (EV), have been solubilised in diblock copolymer micelles, being the design of $A B$ block copolymers based on poly(ethylene glycol) (PEG), as hydrophilic domain, and poly(propylene sulfide) (PPS), as hydrophobic domain ${ }^{2}$. Polymeric micelles, have been obtained from selected polymer systems by the cosolvent displacement method and by a polymer suspension in hot water. Both of the drugs showed a solubility of $2 \mathrm{mg} / \mathrm{mL}$ in aqueous media by PEG-b-PPS micelles and only the $10 \%$ of the encapsulated was released after $24 \mathrm{~h}$

We report here the design and characterization of self-assembling diblockcopolymers, their aggregates characterization and their efficiency in loading drugs and the rate of the spontaneous release, demonstrating that PEG-bPPS systems can successfully be employed as hydrophobic drug carriers.

[1] H. M. Aliabadi, A. Mahmud, A. D. Sharifabadi, A. Lavasanifar, Journal of Controlled Release 2005, 104, 301-311

[2] S.Cerritelli, A.Fontana, D.Velluto, M.Adrian, J.Dubochet, P.De Maria, J. A.Hubbell, Macromolecules 2005, 38, 7845-7851

\section{Polymer Science}

Charge transfer kinetics across modified electrostatic assemblies of polyelectrolytes and metal nanoparticles

\section{Christopher R. Bradbury, Jianjun Zhao \& David J. Fermín}

University of Bern, Department of Chemistry and Biochemistry, Freiestrasse 3, 3012 Bern, Switzerland

The understanding and manipulation of charge transfer across nanostructured interfaces is a key step on the development of novel hybrid optoelectronic and sensing devices. One of the most versatile methods for creating sophisticated nanoscopic architectures is the so-called electrostatic layer-by-layer (LBL) assembly [1]. Despite numerous reports on the electrochemistry of ultrathin films created by LBL, the basic phenomena underpinning the dynamics of electron transport in these systems are not wellunderstood. In this contribution we will look at two different approaches to constructing the LBL film and compare the electron transfer kinetics across the hybrid heterostructure. The first approach is to vary the length of the Self Assembled Monolayer (SAM), followed by the electrostatic attachment of poly-L-lysine (PLL) and citrate stabilised gold nanoparticles. The second approach is to build electrostatic multilayer films of PLL and poly-Lglutamic acid (PGA) terminated with gold nanoparticles and convert the electrostatic assembly into a covalently bonded film. From these two approaches we have shown that the supporting SAM has a significant contribution to the charge transfer kinetic in the absence of nanoparticles. The electrostatic adsorption of gold nanoparticles opens a new path for electron transfer which appears independent of the film thickness. However a strong distance dependence of the electron transfer kinetics between the nanoparticle and the electrode is observed upon crosslinking the LBL film. These results illustrate the ability to control the interactions to favour desired charge transfer pathways and has important implication for the development of hybrid electronic devices.

[1] Decher D., Science, 1997, 277, 1232.

\section{Order creation by rod addition in a $\pi$-conjugated rod-coil block co-} polymer

Nicolas Sary, Cyril Brochon, Georges Hadziioannou, Raffaele Mezzenga

Dept. of Physics, Univ. of Fribourg, Chemin du Musée 3, CH-1700 Fribourg, Switzerland

The morphology arising from the self assembly of rod-coil $\pi$-conjugated poly(diethylhexyloxy-p-phenylene vinylene)-b-poly(styrene) (PPV-b-PS) block copolymers can be tuned to an uncommon extent by the addition of PPV homopolymer. A well-ordered lamellar phase (image b) can be obtained from poorly-structuring PS-b-PPV (image a), and the formation of lamellar clusters can even be induced in a block polymer which on its own shows isotropic ordering. This behaviour is very different from the well known, but limited, phase tuning which can be achieved in coil-coil block copolymers. The study of PPV and PS domain widths shows that the clusters are organized in a smectic $\mathrm{C}$ configuration, characteristic of the weakly segregated regime. The rod-to-rod interaction proves to be stable well above the order-disorder transition temperature, suggesting that the aggregation of the rods is mediated by $\pi-\pi$ interactions and the clusters are thermodynamically stable structures. Based on these observations, we propose a molecular and energetic model giving an account for this driving force toward structure formation.

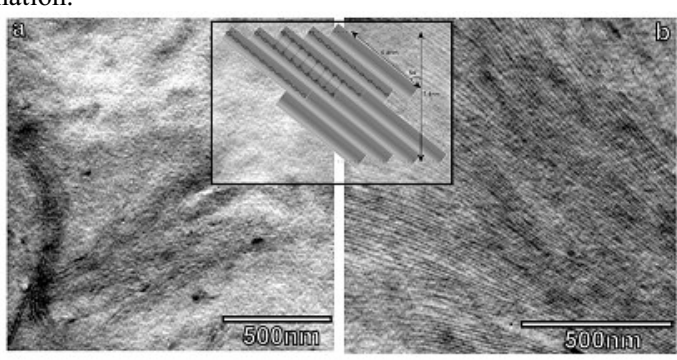

Polymer Science

Fabrication of Organic Light-Emitting Devices by Laser-Induced Forward Transfer Based on Aryltriazene Photopolymer Release Layers

Matthias Nagel ${ }^{\mathrm{a}}$, Romain Fardel ${ }^{\mathrm{a}}$, Pascal Feurer ${ }^{\mathrm{a}}$, Frank Nüesch'; Thomas Lippert $^{\mathrm{b}}$, Alexander Wokaun ${ }^{\mathrm{b}}$

${ }^{\text {a }}$ Laboratory for Functional Polymers, EMPA - Swiss Laboratories for Materials Testing and Research, Überlandstrasse 129, 8600 Dübendorf, Switzerland; ${ }^{\text {b }}$ Paul Scherrer Institut, General Energy Research Demartment, 5232 Villigen PSI, Switzerland

Tailor-made photopolymers containing UV-decomposible aryltriazene chromophores have been synthesized [1] and were applied as UV-absorbing sacrificial release layers for a novel laser-based direct-writing process. Upon pulsed UV-laser irradiation the photopolymer films decompose cleanly in small fragments, generating a photo-triggered pressure-jet which propels an overlaying film of transfer material towards a receiver substrate. This process allows a precise deposition of small pixels of various materials with micrometer resolution. The potential of the novel photosensitive sacrificial release layers was demonstrated recently: Assisted by a $100 \mathrm{~nm}$ thick photopolymer film viable mammalian neuroblast cells were gently transferred onto a bioreceiver substrate [2]. In a similar manner, laterally well-resolved arrays of multispectral nanocyrstal quantum dots (NCQD) were successfully transferred [3]. For the fabrication of light-emitting devices (LED) thin films of semiconducting and electroluminescent polymers have to be deposited with high precision. We show the latest results of our successful laser transfer experiments based on our photofunctional aryl triazene polymers.

[1] M. Nagel, R. Hany, T. Lippert, M. Molberg, F. A. Nüesch, D. Rentsch, Macromol. Chem. Phys. 2007, 208, 277.

[2] A. Doraiswamy, R. J. Narayan, T. Lippert, L. Urech, A. Wokaun, M. Nagel et al. Appl. Surf. Sci. 2006, 252, 4743 - 4747

[3] J. Xu, J. Liu, D. Chui, M. Gerhold, A. Y. Wang, M. Nagel, T. K. Lippert, Nanotechnology 2007, 18, 025403. 
Polymer Science

Molecular Level Control over Hierarchical Structure Formation and Polymerizability of Oligopeptide Substituted Diacetylenes

\section{E. Jahnke, J. Weiss, P. Kreutzkamp, N. Severin, J. P. Rabe, H. Frauenrath*}

ETH Zürich, HCI H520, CH-8093 Zürich, Schweiz

We have successfully prepared hierarchically structured conjugated polymers by a new strategy that can be paraphrased as "self-assemble into a hierarchical structure, then polymerize under retention of the latter" ${ }^{[1]}$. For this purpose, we used diacetylene macromonomers based on $\beta$-sheet forming oligopeptide-polymer conjugates to produce well-defined supramolecular polymers with a finite number of strands in organic solution. These supramolecular polymers gave rise to different superstructures such as single stranded tapes, double-stranded helical ribbons, or quadruple-stranded helix bundles, depending on the arrangement of $\mathrm{N}-\mathrm{H} \cdots \mathrm{O}=\mathrm{C}$ type hydrogen bonding sites in the macromonomers. The precise control of the secondary and higher structure formation allowed to design macromonomers appropriate for a topochemical diacetylene polymerization within the self-assembled aggregates. Thus, the supramolecular polymers were converted into conjugated polymers under retention of their hierarchical structures which could be imaged and manipulated using scanning force microscopy.

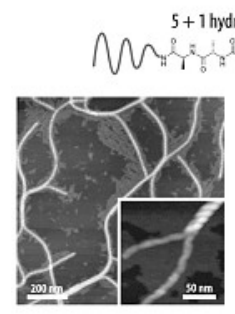

+1 hydrogen bonds
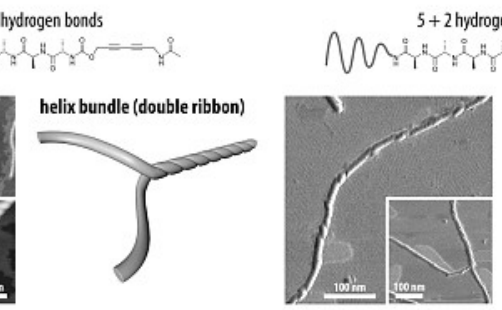

$5+2$ hydrogen bonds

[1] E. Jahnke, I. Lieberwirth, N. Severin, J. P. Rabe, H. Frauenrath, Angew. Chem.Int. Ed. 2006, 45, 5383-5386.

\section{Polymer Science}

Temperature responsive polymers for smart clothing application

$$
\text { Daniel Crespy*, René Rossi }
$$

Empa, Swiss Federal Laboratories for Materials Testing and Research, Lerchenfeldstrasse 5, CH-9014 St. Gallen, Switzerland

Some temperature responsive polymers undergo a coil to globule reversible transition at a certain temperature called the lower concentration solution temperature (LCST). The polymer chains that are in solution below the LCST collapse when the temperature is increased above the LCST.

The $\operatorname{poly}(N$-vinylcaprolactam $)$ displayed below is a temperature biocompatible polymer with a LCST around $32-33{ }^{\circ} \mathrm{C}$, i.e. in the physiological range.

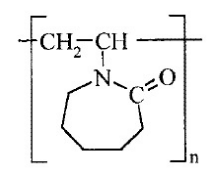

There is an increasing interest in the design of smart stimuli-responsive clothing, especially when the stimulus is the temperature [2]. Smart clothing were fabricated via the coating of textiles with solutions of poly $(N$ vinylcaprolactam). The water vapor transmission rate of the textiles was measured at different temperatures. It was shown that the textiles displayed a temperature responsive permeability.

[1] A. Laukkanen, L. Valtola, F. M. Winnik, H. Tenhu Macromolecules 2004, 37, 2268.

[2] D. Crespy, R. Rossi, Polym. Int. 2007, in press.
Polymer Science

410

Nucleo-copolymers: oligonucleotide-based amphiphilic diblock copolymers

Francisco Teixeira Jr., Cedric Cattin, Markus Ledergerber, Axel Müller, Corinne Vebert-Nardin

University of Basel, Klingelbergstr. 80, 4056 Basel, Switzerland

Nucleo-copolymers are amphiphilic copolymers which have as hydrophilic segment an oligonucleotide sequence ${ }^{1}$. These copolymers take advantage of the high specificity and functionality presented by nucleic acid sequences, which may be considered as negatively charged polymers, in order to build functional macromolecular nanostructures. These nanostructures are also capable of undergoing non-covalent, yet highly specific interaction by basepairing, as well as being recognized by biological systems $\mathrm{s}^{2,3}$. The synthesis of these nucleo-copolymers was performed using different 12-nucleotide long sequences, namely, Guanosine $\left(\mathrm{G}_{12}\right)$, Cytidine $\left(\mathrm{C}_{12}\right)$ and GuanosineAdenosine $\left(\mathrm{A}_{5} \mathrm{G}_{7}\right)$, bearing a terminal carboxylic group, coupled to aminoterminated polybutadiene $(\mathrm{PB}, \mathrm{Mw}=2000 \mathrm{Da})^{4}$. The copolymers were characterized by FT-IR and their self-assembly into vesicular structures in dilute aqueous solution was studied and described through LS and Microscopy analysis. Preliminary biological studies using macrophages show that the self-assembled structures are internalized by the cells and remain stable during this process.

[1] Francisco Teixeira Jr., Per Rigler, Corinne Vebert-Nardin, Chem. Commun. 2007, 1130-1132.

[2] R. A. Chandra, E. S. Douglas, R. A. Mathies, C. R. Bertozzi, and M. B. Francis, Angew. Chem. Int. Ed. 2006, 45, 896-901.

[3] P. Broz, S. M. Benito, C. Saw, P. Burger, H. Heider, M. Pfisterer, S. Marsch, W. Meier, and P. Hunziker, J. Controlled Release 2005, 102, 475-488.

[4] Sergey Nosov, Holger Schmalz, Axel H.E. Müller, Polymer 2006, 47, 4245-4250.

Polymer Science

\section{Synthesis and Application of Polymer modified Nanoparticles}

Andreas Muehlebach*, M. Frey, T. Giesenberg and F. Rime

Ciba Specialty Chemicals Inc., Group Research and R\&D CE Klybeckstrasse, CH-4002 Basel, Switzerland

The physical properties of bulk polymers or coatings can be improved by addition of inorganic nanoparticles while other properties like transparency or fracture toughness are not influenced too much. E.g. higher scratch resistance is obtained with silica or alumina nanoparticles [1] whereas $\mathrm{TiO}_{2}$ or $\mathrm{ZnO}$ nanoparticles lead to invisible UV-protection if the particle size is small enough [2]. However, in both cases the efficiency (=effect divided by the amount added) decreases with smaller particle size while transparency increases. In order to avoid agglomeration or even aggregation and to improve the compatibility with the organic matrix, the inorganic nanoparticles have to be modified with organic groups. In general, a polymeric shell is preferred since it gives the best stabilization due to entropic repulsion of the chains (=steric or electrosteric stabilization) [3].

The poster will display the synthesis of silica nanoparticles of different size, modified with taylor-made polymers and added into a two component polyurethane automotive clear coat and a UV-cured acrylate system. The influence of the modified nanoparticles on hardness, scratch resistance, surface properties and weather-O-meter stability of the coatings will be shown. Furthermore, the performance of synthesized and commercially available inorganic UV-absorbers like nano $\mathrm{TiO}_{2}, \mathrm{ZnO}$ and $\mathrm{CeO}_{2}$ will be compared with some of Ciba's commercial organic UV-absorbers like Tinuvin 400 and Tinosorb M (soluble and micronized UV-A's).

[1] H. Wiese, J. Leuninger, F. Tiarks, B. Schuler, Coating Science International. 2006, Book of Abstracts, 40-48.

[2] W. Caseri, Macromol. Rapid Commun. 2000, 21, 705.

[3] C. Auschra, E. Eckstein, A. Mühlebach, M.-O. Zink, F. Rime, Progress in Organic Coatings 2002, 45, 83. 
Polymer Science

Selective shielding of an antioxidant enzyme by encapsulation into polymeric nanocontainers

\section{W. Meier, C.G. Palivan, F. Axthelm}

Departement Chemistry/University of Basel, Klingelbergstr. 80, 4056 Basel, Switzerland

As superoxide radical anion $\left(\mathrm{O}_{2}^{-}\right)$is involved in oxidative stress present in pathobiochemical processes, such as inflammation, it is an important therapeutic goal to eliminate it from the body. In this respect, $\mathrm{Cu} / \mathrm{Zn}$ Superoxide dismutase (Cu/Zn-SOD) could be an efficient drug candidate. However, as it is quickly eliminated from the bloodstream, $\mathrm{Cu} / \mathrm{Zn}$-SOD provides rather modest protection, if any, when administered.

In this work, we encapsulate $\mathrm{Cu} / \mathrm{Zn}$-SOD in polymeric nanocontainers from self-assembling triblock copolymers in order to bypass its low biodisposability. In general, the versatility of polymer chemistry enables the control of the vesicular self assembly as well as membrane properties, such as permeability. Polymeric nanocontainers offer a better applicability due to their higher stability, compared to liposome carriers ${ }^{[1]}$.

The presence of encapsulated Alexa Fluor 488-tagged Cu/Zn-SOD was determined by EPR spectroscopy and fluorescence measurements. In order to investigate if the protein remains active after encapsulation, pulse radiolysis measurements ${ }^{[2]}$ were accomplished.

$\mathrm{Cu} / \mathrm{Zn}$-SOD remains entirely functional in the hollow cavity of the vesicles. The nanocontainers serve as both a selective shield, as we could observe the penetration of superoxide radical anions across the membrane, and provide an environment mimicking the compartmentalisation and the space constraints of a biological cell.

[1] Jubeh, T.T., Antler, S., Haupt, S., Barenholz, Y., Rubinstein, A., Mol. Pharm. 2005, 2(1), 2 .

[2] Michel, E., Nauser, T., Sutter, B., Bounds, P.L., Koppenol, W.H., Arch. Biochem. Biophys. 2005, 439(2), 234.

\section{Polymer Science}

Vesicles and Monolayers from functionalized Amphiphilic Diblock Copolymers: specific Binding to His-tagged Proteins

\section{Rainer Nehring}

University of Basel, Klingelbergstr. 80, 4056 Basel, Switzerland

Amphiphilic block copolymers are unique building blocks in supramolecular polymer chemistry, both for the generation of highly organized, selfassembeld structures as well for the energetic and structural control of material interfaces.[1]

Polybutadiene-block-polyethylenoxide copolymers were made via anionic polymerization and subsequently quenched with molecules bearing active moieties in a one step procedure. The polymers were characterized by NMR, IR and SEC. These polymers, when appropriately designed, form vesicles and monolayers at the air-water and air-solid interface.

The size and structure of the vesicles are determined by microscopy, freezefracture electron microscopy, FCS and dynamic light scattering. The monolayers are investigated by AFM, LB and BAM.

The chemical active end groups offer various options for further functionalizations. The amphiphilic block copolymers are connected to $\mathrm{N}, \mathrm{N}-$ Bis[(tert-butyloxycarbonyl)methyl]-L-lysine tert-Butylester. Deprotection of the Carboxyl-groups and complexation with nickel (II) results in a specific protein linker. These functional groups are verified by NMR and EPR. HIS-tagged proteins are attached to the NTA-linker. The specific binding is analysed by EPR.

Furthermore the size and structures of the block copolymers - NTA-Ni proteins will be investigated by EPR, NMR and FCS.

[1] Förster, S.; Antonietti, M Adv.Mater. 1998, 10, 195
Polymer Science

Solid supported block copolymer membranes:

A new platform in sensing technology?

Serena Belegrinou, Rainer Nehring, Violeta Malinova, Katarzyna Kita-Tokarczyk, Wolfgang Meier

University of Basel, Department of Chemistry, Klingelbergstrasse 80, 4056 Basel, Switzerland

For more than 20 years, solid supported membranes, formed by selfassembly of phospholipids on solid substrates, have been most commonly used to mimic cell surfaces. ${ }^{[1]}$ However, the use of solid supported membranes bears essential disadvantages. As the membranes are often deposited directly on the bare solid support, the integrated transmembrane proteins undergo undesired denaturation processes. To prevent the proximity of the artificial membranes and the substrate, and thus to increase the protein mobility, soft polymeric materials can be used as spacer units. ${ }^{[2]}$ The combination of biological and artificial materials offers great potentials for biotechnological applications, e.g. a unique platform for highly selective sensing devices. Over the last years, amphiphilic block copolymers have aroused particular interest. The replacement of phospholipids by block copolymers enables the design of thicker, less permeable, and mechanically and chemically more stable membranes. In particular, the robustness against air is a crucial advantage of planar tethered block copolymer membranes. ${ }^{[3]}$

In order to achieve this aim, amphiphilic block copolymers have been functionalized with terminal thiol groups to enable the immobilization on gold surfaces. We will present different synthetic approaches and a characterization of the membrane properties depending on polymer chemistry and block ratio, as well as membrane preparation methods.

[1] Tanaka, M.;Sackmann, E., Phys. Stat. Sol. (a) 2006, 203, 3452.

[2] Tanaka, M.;Sackmann, E., Nature 2005, 437, 656.

[3] Taubert, A.;Napoli, A.;Meier, W., Curr. Opin. Chem. Biol. 2004, 8 , 598.

Polymer Science

\section{Fractal Dimension and Localization of DNA Knots}

Erika Ercolini ${ }^{1}$, Francesco Valle ${ }^{1}$, Jozef Adamcik ${ }^{1}$, Guillaume Witz ${ }^{1}$, Ralf Metzler $^{2}$, Paolo De Los Rios ${ }^{3}$, Joaquim Roca ${ }^{4}$, and Giovanni Dietler

' Ecole Polytechnique Fédérale de Lausanne (EPFL), Laboratory of Physics of Living Matter, IPMC, CH-1015 Lausanne, Switzerland

${ }^{2}$ Nordic Institute for Theoretical Physics (NORDITA), Bledamsvej 17, 2100 Copenhagen OE, Denmark

${ }^{3}$ Ecole Polytechnique Fédérale de Lausanne (EPFL), Laboratory of Statistcal Biophysics, ITP, CH-1015 Lausanne, Switzerland

${ }^{4}$ Department of Molecular Biology, CID-CSIC, 08034 Barcelona, Spain

The scaling properties of homo- or heterogeneous mixture of DNA knot types were studied by Atomic Force Microscopy (AFM) [1]. DNA knots were adsorbed onto freshly cleaved mica following two different protocols: (i) strong binding (deposition on 3-aminopropyltriethoxy silane vapors modified mica) that induces a kinetic trapping of the three-dimensional (3D) configuration, and (ii) weak binding (deposition from a solution containing $\mathrm{Mg}^{2+}$ ions) that permits partial relaxation on the surface. The fractal dimension was determined from the AFM images using the box counting algorithm calculating the number of boxes $\mathrm{N}(\mathrm{L})$ containing a part of the molecule as a function of the box size L. In (i) the radius of gyration of the adsorbed DNA knot scales with the 3D Flory exponent $v \approx 0.60$ within error, a result in very good agreement with the renormalization group prediction for linear self-avoiding polymers in $3 \mathrm{D}$. In (ii), we find $v \approx 0.66$, a value between the 3D and 2D $(v=3 / 4)$ exponents. From the AFM images of weakly adsorbed simple knots we can deduce the localization behavior, which was predicted for 2D self avoiding chains [2]

[1] E. Ercolini, F. Valle, J. Adamcik, G. Witz, R. Metzler, P. De Los Rios, J. Roca, and G. Dietler, Phys. Rev. Lett. 2007, 98, 058102

[2] R. Metzler, A. Hanke, P. G. Dommersnes, Y. Kantor, and M. Kardar Phys. Rev. Lett. 2002, 88, 188101. 
Polymer Science

\section{Ionic Liquid Assisted Polymerization Reaction}

Tilmann J. Geldbach, Amira Abou-Hamdan

C. Guerrero-Sanchez, T. Erdmenger, P. Šereda, D. Wouters, U. S. Schuber

Chemspeed Technologies, Augst, Switzerland DPI, Eindhoven, NL

Heterogeneous polymerization can be defined as a reactive liquid-liquid dispersion in which the nature of the suspended drops changes as the polymerization reaction proceeds. Common suspension polymerizations are carried out in stirred tank reactors, to which a suspending agent (protective colloid) is added to the aqueous phase in order to keep the drops from coalescing as they change from the liquid to the solid state.

Of major importance for the stability of suspensions is the type and amount of suspending agent utilized, due to the fact that it induces electrostatic charges on the surface of the suspended monomer-polymer particles, which retard their coalescence. In this contribution water-soluble ionic liquids (WSIL) are proposed as reaction media and as alternative stabilizers in suspension polymerizations. Ionic liquids (IL) are of great interest in these applications due to the fact that their properties can easily be tailored to meet specific needs and thereby allow for the synthesis of well-defined polymer beads with narrow size distributions.

The use of IL as surfactants and as a reaction media in suspension polymerizations allows for the tuning of the size of polymer beads and other of their characteristics. Even though suspension polymerization is a highly complex polymerization process, the synthesis of several polymeric materials by this synthetic approach as well as the screening of their reaction parameters can easily be adapted to be performed in a parallel-automated manner.

[1] C. Guerrero-Sanchez, T. Erdmenger, P. Šereda, D. Wouters, U. S. Schubert, Chem. Eur. J. 2006, 12, 9036

\section{Polymer Science}

Chiral liquid-crystalline "Janus-type" fullerodendrimers: synthesis and characterization

Thi Nhu Y Hoang, Cyril Kopp, Natacha Maringa and Robert Deschenaux*

Institut de Chimie, Université de Neuchâtel, Avenue de Bellevaux 51, Case postale 158, 2009 Neuchâtel, Switzerland

E-mail:thi.hoang@unine.ch,cyril.kopp@unine.ch

A new class of "Janus" liquid crystal macromolecules has been prepared from two dendrons displaying different liquid crystal properties. The ramified poly(benzyl ether) dendron ${ }^{[1]}$ and the aliphatic ester dendron ${ }^{[2]}$ present cholesteric and chiral smectic phases, respectively. From the point of view of supramolecular chemistry, it is of interest to study the mesomorphic properties as well as the organization of such heterodendrimers.

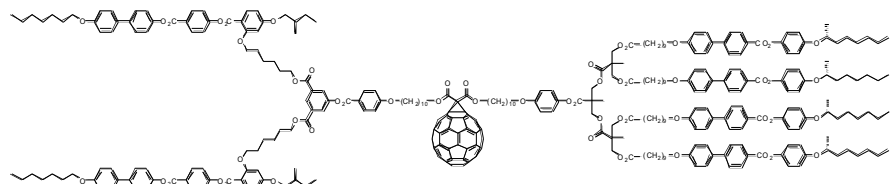

Fullerodendrimers have been synthesized from the above malonates via the Bingel reaction. ${ }^{[3]}$ Their liquid-crystalline properties have been studied to explore the influence of each dendron (generation, nature, mesogenic groups) on the supramolecular organization within the liquid-crystalline phases. The combination of fullerene with chiral dendrimers open the doors for the design of functional ferroelectric materials.

[1] S. Campidelli, C. Eng, I. M. Saez, J. W. Goodby, R. Deschenaux, Chem. Commun., 2003, 1520

[2] P. Busson, J. Örtegren. H, Ihre, U. W. Gedde, G. Andersson, A. Hult, Macromolecules, 2001, 34, 1221.

[3] C. Bingel, Chem. Ber., 1993, 126, 1957
Polymer Science

Polymerization of novel highly charged cationic monomers: Kinetic basic study and solution behavior of homopolymers

\section{R. Losada, C.Wandrey}

Ecole Polytechnique Fédérale de Lausanne, Institut de Bioingénierie, Laboratoire de Médecine Régénérative et de Pharmacobiologie, Lausanne, Switzerland.

The importance of cationic water soluble polymers has increased for many years, due to the widespread application fields. The majority of new polyelectrolyte structures has been obtained by polymer modification or copolymerization. Only a few new polyelectrolyte structures have been synthesized by polymerization of new monomers.

We present research on novel polyelectrolyte structures obtained from two monomers: bis-1,3(N,N,N-trimethylammonium)-2-propylmethacrylate dichloride (dipole $\mathrm{M}$ ) and 1,3(N,N,N-trimethylammonium)-2-propylacrylate dichloride (dipole A) possessing two cationic charges per acrylic monomer unit.

The kinetics of azo cationic initiated polymerization of dipole $\mathrm{M}$ in solution was investigated. The monomer and initiator exponents of the overall polymerization rate equations were obtained as 4.40 and 0.59 , respectively. The polymer solution behavior was characterized by dilution viscometry. As expected the homopolymers behave as very strong polyelectrolytes in aqueous solution presenting significant differences of the reduced viscosities obtained in water and aqueous solution with addition of low molar mass salt. Strong polyelectrolyte characteristics were confirmed by counterion activity measurements yielding counterion activity coefficients as low as 0.11 . Such low values are predicted by polyelectrolyte theories for a charge distance of $0.12 \mathrm{~nm}$ realized in the homopolymers

Acknowledgements: The work was supported by the Swiss National Science Foundation, grant 200021-107737. The monomers were provided by TAMINCO.

Polymer Science

\section{Study of the Rod-to-Coil Transition in Polypeptide / $\pi$-Conjugated / Polypeptide Triblock Copolymers}

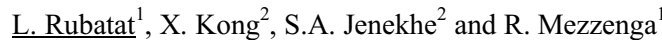

${ }^{1}$ Fribourg University, Physics Dept., Fribourg, Switzerland ${ }^{2}$ University of Washington, Chem. \& Engineering. Dept, Seattle, USA

We investigate the bulk ordering of a series of novel triblock copolymers (BLGm-HFn-BLG $m$ ) containing poly(9,9-dihexylfluorene-2,7-diyl) (PHF) and poly $(\gamma$-benzyl-L-glutamate) (PBLG) (Cf. Figure 1). These samples, prepared in Jenekhe's lab, [1] are the first examples of hybrid copolymers containing $\pi$-conjugated polymers and polypeptides. They can be employed in several applications such as biosensors, scaffolds for tissue engineering and nanoelectronics. The copolymers exhibit two different conformations: rodrod-rod and coil-rod-coil by virtue of the rod-to-coil transition of the polypeptidic helix. Three copolymers with different block ratio $(\mathrm{m} / \mathrm{n}: 23 / 15$, $54 / 15$ and 16/28) are examined in both conformations using small and wide angle scattering techniques (SAXS and WAXS) correlated with Transmission Electron Microscopy. The morphologies and the $\pi$-conjugated PHF confinement effect on the UV-vis absorption spectra are discussed.

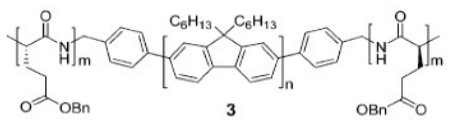

Fig. 1. (BLGm-HFn-BLGm) tribloc chemical structure 
Polymer Science

\section{Architectural backbone influence on ionic liquid crystalline comb-like} polymers

Nadia Canilho ${ }^{1}$, Markus Scholl ${ }^{2}$, Edis Kasëmi ${ }^{3}$, Harm-Anton Klok ${ }^{2}$, Dieter Schlüter ${ }^{3}$, Raffaele Mezzenga ${ }^{1^{*}}$

${ }^{1}$ Department of Physics and Fribourg Center for Nanomaterials, University of Fribourg, Ch. du Musée 3, Perolles, CH-1700 Fribourg (Switzerland)

${ }^{2}$ École Polytechnique Fédérale de Lausanne, Institut des Matériaux Laboratoire des Polymères, CH-1015 Lausanne, Switzerland

${ }^{3}$ Department of Materials, Swiss Federal Institute of Technology (ETHZ), Wolfgang Pauli Strässe 10, 8093 Zurich, Switzerland

Ionic self-assembly between polyelectrolyte polymers and ionic mesogens is a simple way to design new supramolecular structures whose phase diagram depends directly on the molecular architecture of the macromolecular templates. For that, cationic polyelectrolytic hyperbranched polymers (HBP) and dendronized polymers of three generations (DPGx) were used to form the backbone of new reversible ionic comb-like polymers complexes [1],[2] A range of $\mathrm{C} 8$ to $\mathrm{C} 18$ hydrocarbon alkyl tails were chosen as oppositely charged mesogenes to be complexed with branched polymers. As a consequence of the different chemical nature, the two building blocks have a tendency to microphase separate and self-assemble into various liquid crystalline phases. These nano-structured phases can be reached by controlling the architecture of the polyelectrolyte and the length of the mesogene. Small angle x-ray scattering (SAXS), cross polarized optical microscopy (CPOM), and differential scanning calorimetry (DSC) techniques revealed that ionic complexes of HBP and DPGx in the solid state shown columnar hexagonal, rectangular and tetragonal phases or lamellar arrangement with a lattice parameter ranging between of 3 to $5 \mathrm{~nm}$, depending on the length of the hydrocarbon chain of the anionic surfactant and the dendritic generation.

[1] Canilho N., Scholl M., Mezzenga R., Klok A.-H., in preparation.[2]

Canilho N., Kasëmi E., Schlüter A.D., Mezzenga R., Macromolecules 2007, 40, 2822

\section{Polymer Science}

Peptide-synthetic hybrid block copolymers and their surfactant complexes: solid state behavior

Matthew R. Hammond $\neq$, Harm-Anton Klok $\uparrow$, and Raffaele Mezzenga $\ddagger$

† Department of Physics, University of Fribourg, Chemin du Musée 3, 1700 Fribourg, Switzerland

† École Polytechnique Fédérale de Lausanne, Institut des Matériaux Laboratoire des Polymères, CH-1015 Lausanne, Switzerland

We report on the solid-state nanostructures formed in ionic supramolecular complexes based on a series of poly(L-glutamic acid)-b-poly(ethylene oxide) (PLGA-PEO) peptide-synthetic block copolymers. The complexes can be formed by two methods of ionically grafting alkyl chains to the carboxylic acid side groups of the PLGA blocks: (a) by complexation with trimethylalkylammonium surfactants in aqueous media, or (b) with primary alkylamines via acid-base proton exchange and subsequent ionic bonding in organic solvents. Complex formation is monitored by NMR and FTIR, and solid state structural results are obtained from small- and wide-angle x-ray scattering (SAXS/WAXS) and transmission electron microscopy (TEM).

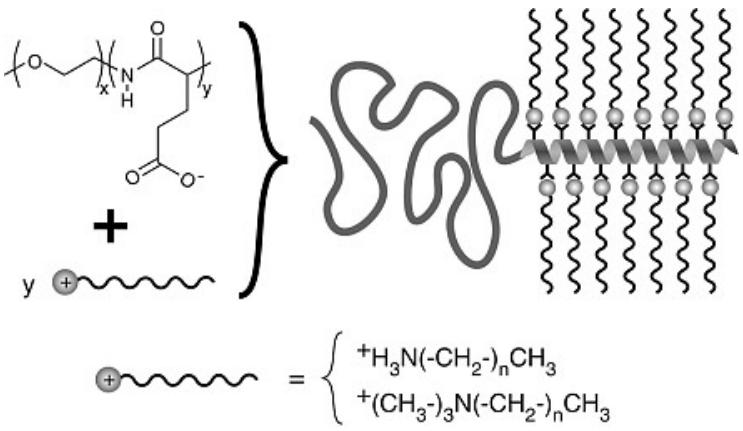

Polymer Science

Heterobifunctional Poly(2-methyl-2-oxazoline) for the Fabrication of Functional Non-Fouling Surface Coatings via 'Click Chemistry'

Rupert Konradi ${ }^{\#}$, Qiguang Li ${ }^{\#}$, Marcus Textor ${ }^{\#}$ Erich Nyfeler ${ }^{\S}$, Andreas Mühlebach ${ }^{\S}$, Niklaus Bühler ${ }^{\S}$

"Laboratory for Surface Science and Technology, Department of Materials, ETH Zurich, Wolfgang-Pauli-Strasse 10, 8093 Zürich, Switzerland ${ }^{\S}$ Ciba, Group Research, Klybeckstrasse 141, CH-4002 Basel, Switzerland

We have recently investigated poly(2-methyl-2-oxazoline) (PMOXA) as an alternative polymer to poly(ethylene glycol) (PEG) for the fabrication of non-fouling surface coatings. ${ }^{1}$ Like PEG this polymer is nonionic and hydrophilic having a biomimetic 'pseudopeptide' structure. Surfaces coated with graft copolymers of poly(L-lysine) (PLL) with PMOXA side-chains (PLL-g-PMOXA) showed excellent protein and bacteria repellent properties. We are now striving to equip these graft copolymers with a bioconjugation site for the attachment of biologically active functions. This will allow us to specifically control the interfacial biological response on a bioinert polymeric support. We describe the synthesis of alkynyl-terminated PMOXA by use of a novel alkynyl-functional initiator for the living cationic polymerization of 2-methyl-2-oxazoline. The propargyl terminal group will allow for the copper(I)-catalyzed cycloaddition of azide-functionalized biological moieties ('click chemistry') (see Figure 1).
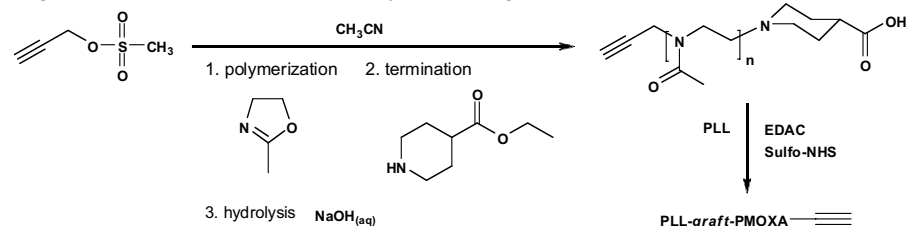

Figure 1. Synthesis of alkynyl-functional PLL-g-PMOXA

[1] Konradi, R., Pidhatika, B., Textor, M. in preparation

\section{Polymer Science}

“Protein-multi-catalyst" biohybrids: Synthesis and activity studies.

Benjamin Le Droumaguet, Sidhanath Bhosale, Sylvain Koeller, and Kelly Velonia

University of Geneva, Sciences II, CH-1211 Geneva 4, Switzerland.

The specific functionalization of proteins aiming in the formation of welldefined nanoscaled structural and functional devices has been in the forefront of chemical research during the last decades [1].
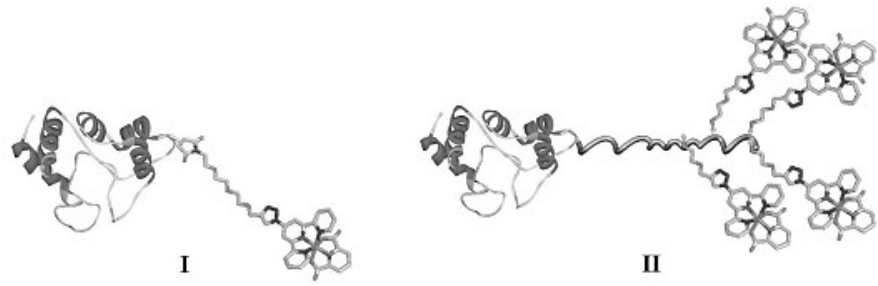

Targeting such multifunctional biohybrids, we synthesized novel proteinmetallo-organic catalyst adducts (I). Interestingly, these adducts showed to retain the catalytic activity of both the protein and the organic catalyst component. Aiming at even more efficient catalysts, we also present here early results on the synthesis, the combined catalytic activity and aggregation behaviour of multicatalytic triblock protein-polymer hybrids (II) which were synthesized using the "post-functionalization" approach [2].

[1] P. Thordarson, B. Le Droumanguet, K. Velonia J. App. Microbiol. Biotechnol. 2006, 73, 243.

[2] B. Le Droumaguet, G. Mantovani, D.M. Haddleton, K. Velonia, J. Mater. Chem. 2007, in press DOI: 10.1039/b618079e. 
Polymer Science

Monte Carlo simulations of polyelectrolyte chains and colloids with explicit counterions

\section{F. Carnal, S. Stoll}

Department of Inorganic, Analytical and Applied Chemistry University of Geneva

Quai Ernest-Ansermet 30, 1211 Geneva 4, Switzerland

The complexation of polyelectrolyte chains with oppositely charged colloids has recently attracted much attention, because of their important potential applications in nanoscience, environmental chemistry and biology. Polyelectrolyte chains are usually associated with a large range of compounds, including oppositely charged polymers, colloids (inorganic particles, surfactant micelles) and biomacromolecules (proteins, DNA)

A common way to understand physico-chemical properties between polyelectrolyte chains and colloids is to perform Monte Carlo simulations according to the Metropolis algorithm.

Using Monte Carlo simulations, we investigate the case of an isolated polyelectrolyte chain and a polyelectrolyte chain in presence of colloids. The counterions in the solution are described explicitly using the primitive model of electrolytes. The interactions between particles are assumed to be pairwise additive and the solvent enters the model only through its relative permittivity. We focused on the influence of counterions on the conformations and on the physico-chemical properties of the polyelectrolyte chain and colloids in the system. For that purpose, radial distribution and entropy functions calculated.

\section{Polymer Science}

\section{Vesicles as Templates for the Enzymatic Synthesis of Polymers}

\section{Zengwei Guo, Peter Walde*}

Department of Materials, ETH Zürich, Wolfgang-Pauli-Str. 10, CH-8093 Zürich, Switzerland

Enzyme-catalyzed polymerization reactions are potentially environmentally friendly processes [1]. One example is the use of aniline and aniline derivatives and peroxidase to prepare conducting polyaniline [2-4]. Depending on the chemical structure of the monomers, the presence of templates promotes the formation of the para-linked form of the polymers. The templates used so far include negatively charged polymers [4] and surfactant micelles [2, 4]. In the case of aniline, peroxidase-catalyzed polymerization leads to the formation of mainly linear polymers, while in the absence of templates, highly branched products are obtained [2-4].

Preliminary measurements have shown that negatively charged vesicles can be applied as templates as well [5]. In a series of systematic measurements, the polymerization of aniline has been studied under different experimental conditions. VIS/NIR absorption spectra indicate that vesicles - which have a much lower curvature as compared to micelles - may even serve as better templates than micelles to obtain linear polyaniline chains.

[1] M. Reihmann, H. Ritter, Adv. Polym. Sci. 2006, 149, 1 .

[2] W. Liu, J. Kumar, S. Tripathy, L. A. Samuelson, Langmuir 2002, 18, 9696

[3] S.-C. Kim, P. Huh, J. Kumar, B. Kim, J.-O. Lee, F. F. Bruno, L. A. Samuelson, Green Chem. 2007, 9, 44.

[4] P. Xu, A. Singh, D. L. Kaplan, Adv. Polym. Sci. 2006, 194, 69.

[5] T. Namani, P. Walde, Langmuir 2005, 21, 6210.
Polymer Science

\section{Polymer and Nanoparticle Complex formation}

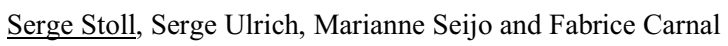

University of Geneva,

Department of Inorganic, Analytical and Applied Chemistry, Analytical and Biophysical Environmental Chemistry (CABE) Sciences II, 30 quai E. Ansermet, CH - 1211 Geneva 4, Switzerland

Electrostatically induced interactions between nanoparticles and charged polymers (polyelectrolytes) and the resulting complex formation are expected to find important issues (gene therapy, bioengineering, transport of trace pollutants) as well as in industrial applications (purification of water as flocculating water insoluble mixtures). The long range attractive and/or repulsive character of electrostatic interactions between charged polymers and nanoparticles give these complexes specific properties which are partially understood. Owing to the important potential of computer simulations to provide qualitative and quantitative means of understanding the factors that influence polyelectrolytes and nanoparticles interactions, a Monte Carlo approach is used here to get an insight into the behaviour of a weak polyelectrolyte with the presence of oppositely charged nanoparticles. The adsorption/desorption limit as well as the total number of adsorbed nanoparticles which are key parameters for applications in water treatment of polyelectrolyte/nanoparticle mixtures and to control nanoparticle chemical reactivity are also investigated.

[1] The many facets of polyelectrolytes and oppositely charged macrions complex formation", S. Ulrich, M. Seijo, S. Stoll, Current Opinion in Colloid \& Interface Science, 2006, 11, 268.

Polymer Science

Towards High-k Silicone Composite Material for Low-Voltage Dielectric Elastomer Actuators

Dorina Opris ${ }^{1}$, Martin Molberg ${ }^{1}$, Christiane Löwe' ${ }^{1}$, Frank Nüesch ${ }^{1}$, Yves Leterrier ${ }^{2}$, Christopher Plummer ${ }^{2}$, Jan-Anders Månson ${ }^{2}$

'Empa, Swiss Federal Institute for Materials Testing and Research, Ueberlandstrasse 129, CH-8600 Dübendorf; ${ }^{2}$ École Polytechnique Fédéral de Lausanne, EPFL-IMX-LTC Station 12, CH-1015 Lausanne

The characteristics of electroactive polymers (EAP) to emulate biological muscles made them attractive for various applications. Of growing interest in the field of EAP are the dielectric elastomer actuators (DEA) for their ability to produce large strains ( $>200 \%)$ and relatively simple working principle. However, a broad application of DEA is hindered by their high driving voltage in the $\mathrm{kV}$ regime. To lower this driving voltage there are two parameters that can be changed: lower the thickness of the actuator film and increase the dielectric constant of the material [1].

We have shown that blending of high-k filler material like oligo-copper phthalocyanine into a silicone matrix enhances the dielectric constant significantly [2]. Better compatibility of the filler with a polyurethane matrix and good dispersion leads to a dramatic ( $>500$-fold) increase of the dielectric constant in this polymer composite and yields in better electromechanical properties, when applied in actuators [3].

Following the effective concept of composite materials, we develop novel silicone composites by filling them with phthalocyanines or conductive particles (metals and conductive polymers). For that purpose we synthesize different phthalocynanines to study the influence of e.g. the aggregation behavior or particle size on the dielectric constant. Preparations of films of such binary systems as well as their physical, mechanical and electromechanical characterization are presented.

[1] R. Pelrine et al., Science 2000, 287, 836.

[2] X. Zhang et al., Adv. Eng. Mat. 2005, 5, 361

[3] Ch. Huang et al., Appl. Phys. Lett. 2005, 87, 182901 
Polymer Science

\section{In Situ Formation of Protein-Polymer Giant Amphiphiles using ATRP} Polymerization.

\author{
Benjamin Le Droumaguet, Kelly Velonia
}

University of Geneva, Sciences II, CH-1211 Geneva 4, Switzerland.

Due to their widespread utility in medical applications, biotechnology, and nanotechnology, polymer-protein conjugates are in the forefront of chemical research. During the last decades they have been prepared by conjugation of functionalized polymer chains to biomolecules through covalent and/or bioaffinity bindings, approaches that often involve multiple steps of synthesis, chemical modification, purification, and conjugation.

A novel strategy eliminating these multistep procedures and leading to welldefined polymer-protein conjugates has recently been introduced [1]. By using appropriately modified proteins as initiating sites for ATRP or RAFT polymerization, a variety of hydrophilic bioconjugates has been synthesized and characterized.

The study presented here extends this concept to the formation of Giant Amphiphiles comprising a protein specifically connected to a hydrophobic (e.g. polystyrene) or multifunctional polymer [2]. With the use of a macroinitiator functionalized protein and the appropriate hydrophobic monomer, we were able to achieve ATRP polymerization starting from the protein. Furthermore, preliminary results show that the degree of polymerization is controlled by the formation of the giant amphiphilic biohybrids and their concurrent aggregation.

[1] K.L. Heredia, D. Bontempo, T. Ly, J.T. Byers, S. Halstenberg, H.D. Maynard, J. Am. Chem. Soc. 2005, 127, 16955. J. Nicolas, V. San Miguel, G. Mantovani, D.M. Haddleton Chem. Commun. 2006, 4697. J. Liu, V. Bulmus, D.L. Herlambang, C. Barner-Kowollik, M.H. Stenzel, T.P. Davis, Angew. Chem. Int. Ed. 2007, 46, 3099.

[2] B. Le Droumaguet, G. Mantovani, D.M. Haddleton, K. Velonia, J. Mater. Chem. 2007, in press DOI: 10.1039/b618079e.

\section{Polymer Science}

A Model for the Hierarchical Self-Organization of Diacetylene Functionalized Oligopeptide Polymer Conjugates

E. Jahnke, J. Weiss, P. Kreutzkamp, N. Severin, J. P. Rabe, H. Frauenrath*

\section{ETH Zürich, HCI H520, CH-8093 Zürich, Schweiz}

We have successfully prepared hierarchically structured conjugated polymers via self-assembly and topochemical polymerization of diacetylene macromonomers based on $\beta$-sheet forming oligopeptide-polymer conjugates. $^{[1]}$ Thus, uniform supramolecular polymers with a finite number of strands and defined superstructures were obtained, such as single-stranded tapes, double-stranded helical ribbons, or quadruple-stranded helix bundles. The superstructural diversity was rationalized to result from the number and arrangement of $\mathrm{N}-\mathrm{H} \cdots \mathrm{O}=\mathrm{C}$ type hydrogen bonding sites in the macromonomers. The precise control over the hierarchical structure formation allowed to design macromonomers appropriate for a topochemical polymerization within the self-assembled aggregates. Accordingly, high molecular weight soluble poly(diacetylene)s with single-stranded, double- or quadruple-helical quaternary structure were produced.

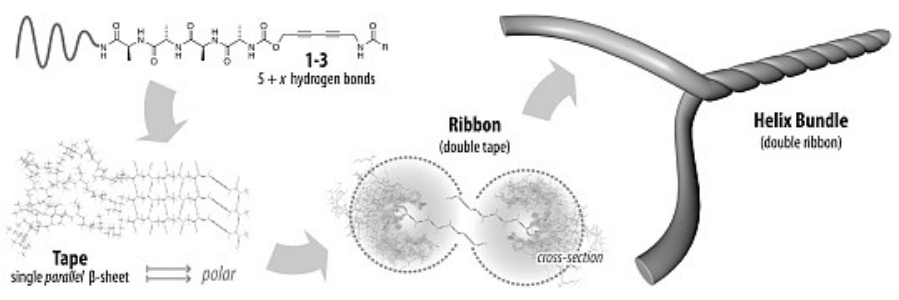

[1] E. Jahnke, I. Lieberwirth, N. Severin, J. P. Rabe, H. Frauenrath, Angew. Chem.Int. Ed. 2006, 45, 5383-5386.
Polymer Science

Cross-linkable Poly(2-methyl-2-oxazoline)-based Polymers for Non-Fouling Hydrogel Coatings

Torben Gillich, Bidhari Pidhatika, Marcus Textor, Rupert Konradi

Laboratory for Surface Science and Technology, Department of Materials, ETH Zurich, Wolfgang-Pauli-Strasse 10, 8093 Zürich, Switzerland

We have recently identified poly(2-methyl-2-oxazoline) (PMOXA) as a promising alternative polymer to poly(ethylene glycol) (PEG) for the fabrication of non-fouling surface coatings. ${ }^{1}$ In analogy to the well-studied poly(L-Lysine)-graft-poly(ethylene glycol) (PLL-g-PEG), graft-copolymers consisting of poly(2-methyl-2-oxazoline) (PMOXA) side-chains grafted onto a polycationic PLL backbone (PLL-g-PMOXA) spontaneously adsorbed onto oppositely charged metal oxide surfaces and rendered them highly resistant to protein and bacterial adhesion. ${ }^{1,2}$

We are now developing novel UV-cross-linkable PMOXA-based polymers for the preparation of non-fouling hydrogel coatings. The hydrogel architecture enables the coating of polymeric and hydrophobically-modified substrates and allows to adjust the coating thickness via process parameters.

Here we present the synthesis of a novel 2-oxazoline monomer that carries a photocross-linkable side-group and its copolymerization with 2-methyl-2oxazoline to obtain both, random and block copolymers. The different copolymers will be applied to surfaces through simple spin- and dip-coating processes followed by UV cross-linking and the resulting hydrogel coatings will be examined with respect to their resistance to protein adsorption.

[1] Pasche, S.; De Paul, S. M.; Voeroes, J.; Spencer, N. D.; Textor, M. Langmuir 2003, 19, 9216-9225

[2] Konradi, R., Pidhatika, B., Textor, M. in preparation

Polymer Science

\section{Cellular response onto oligonucleotide-coated substratum}

Julia Razumovitch, Kelnner Rodrigues de França, Markus Ledeberger, Wolfgang Meier, Corinne Vebert-Nardin

University of Basel, Klingelbergstr. 80, 4056 Basel, Switzerland

Though the hybridization process is ruling the DNA technology, a full understanding of the mechanism has not been achieved yet. Nevertheless, a theoretical description has been addressed very recently by Halperin et al. We thus adopted a physico-chemical approach to assess the outcomes. In this contribution, we will thus present our investigations on the preparation and minute characterization of oligonucleotide-coated surfaces and subsequent hybridization.

A further peculiar aspect of oligonucleotide sequences is their recognition by cells, which by oligonucleotide coating, enables the bio-activation of a substratum. Osteoblasts seeded onto such nucleotide-coated surfaces indeed exhibit a positive cellular response as demonstrated by fluorescence microscopy 
Polymer Science

New Soluble Poly(diacetylene)s Utilizing Perfluorophenyl-Phenyl Interactions as a Supramolecular Synthon

Rui Xu, W. Bernd Schweizer, Holger Frauenrath*

\section{Eidgenössische Technische Hochschule Zürich, Department of Materials Hönggerberg, HCI H515, CH-8093 Zürich, Switzerland}

Research in topochemical polymerizations has recently been reinvigorated by concepts of supramolecular chemistry and crystal engineering. ${ }^{[1]} \mathrm{We}$ have previously successfully utilized $\mathrm{C}_{6} \mathrm{H}_{6}-\mathrm{C}_{6} \mathrm{~F}_{6}$ interactions as a supramolecular synthon in topochemical diacetylene polymerizations and generated the first alternating diacetylene copolymer. ${ }^{[2]}$ Here, we report series of diacetylene monomers, in which $\mathrm{C}_{6} \mathrm{H}_{6}-\mathrm{C}_{6} \mathrm{~F}_{6}$ interactions outperform the packing of long alkyloxy chains in crystallizations and in preorganizing the monomers for topochemical polymerizations. The role of $\mathrm{C}_{6} \mathrm{H}_{6}-\mathrm{C}_{6} \mathrm{~F}_{6}$ interactions in assembling the monomers in crystalline forms was compared, and the correlation with monomer polymerizability was studied in detail. From one of the monomers, a new soluble poly(diacetylene) was obtained, the structure of which was proved by Raman spectroscopy, solution state NMR, as well as a polymer crystal structure.
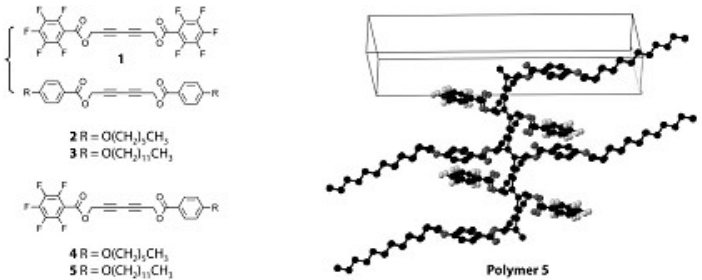

[1] R. H. Grubbs et al. Angew. Chem. Int. Ed. 1997, 36, 248; F. W. Fowler et al. Angew. Chem. Int. Ed. 2000, 39, 2132; MacGillivray et al. Angew. Chem. Int. Ed. 2004, 43, 232.

[2] H. Frauenrath et al. J. Am. Chem. Soc. 2006, 128, 5541.

Polymer Science

\section{Responsive core-shell silica nanoparticles}

\section{Lucy Kind", Axel Müller**, Uwe Pieles ${ }^{* * *}$, Wolfgang Meier}

"University of Basel; Department of Chemistry; Klingelbergstrasse 80; 4056 Basel; Switzerland

${ }^{* *}$ University of Bayreuth; Department of Molecular Chemistry II; Universitätsstrasse 30; 95447 Bayreuth; Germany

${ }^{* * * *}$ Fachhochschule Nordwestschweiz; University of Life Sciences; Department of Chemistry and Bioanalytic; Gründenstrasse 40; 4132 Muttenz; Switzerland

Silica nanoparticles gain increasing interest because of their excellent properties such as low density, high specific surface area, adsorption capacity, and the ability for encapsulation. These characteristics can be combined in a hybrid particle where star shaped polyelectrolytes like $\operatorname{poly}(N, N-$ dimethylaminoethyl methacrylate (poly(DMAEMA)) form a template for the condensation of tetraethyl orthosilicate (TEOS), which leads to the formation of a silica shell around the polymer molecules.

Here we investigated the morphology of the silica nanoparticles. The core shell structure of the particles makes them highly interesting for host / guest encapsulation that is relevant for bioimaging or triggered release. Here it is particularly interesting that the polymer core of the particles shows a $\mathrm{pH}$ and temperature dependant solution behavior. The associated conformational changes of the polymer create a mechanical force on the inorganic shells that can be used as a trigger for controlled release.
Polymer Science

Probing Heparin Functionalization of Polymer Vesicles by Specific Peptide Binding

Diana Sebök, Alessandro Napoli, Benjamin Bircher, Wolfgang Meier

University of Basel, Klingelbergstr. 80, 4056 Basel, Switzerland

Heparin is a linear, negatively charged polysacchride that interacts mainly with positively charged proteins. Due to its anticoagulant activity and interaction with many growth factors, it offers a wide range of pharmacological applications.

We have synthesized amphiphilic copolymers composed of a hydrophobic poly(dimethyl-siloxane) (PDMS) block and LMW heparin as the hydrophilic one. The insertion of such copolymers into the membranes of vesicles formed by PEG-poly(thioethers) diblock copolymers was exploited to biofunctionalize these potential drug carriers. Protamine salmine A1, a small, positively charged peptide with a high binding affinity to heparin was used to verify if heparin coats the vesicle membranes and keeps its biological activity.

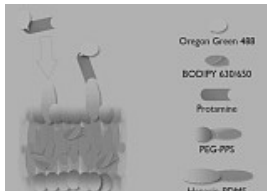

Fig 1: The two fluorescent dyes enable to locate the PEG-PPS membrane and Protamin

The binding of protamine to heparin coated giant vesicles was investigated by laser scanning confocal microscopy and fluorescence (cross-) correlation spectroscopy FCS/FCCS. Results suggest that Hep-PDMS copolymers incorporate at a molar ratio of 1:100 still displaying binding activity.

[1] Napoli et al. Nature Materials, 2004, 3, 183

Polymer Science

Novel screening protocol for universal applicable adhesives to limit surface biofouling

M.Textor, S. Zürcher, S.Tosatti, K.Gademann, S.Saxer

ETH Zürich, Wolfgang-Pauli-str. 10, 8093 Zürich, Switzerland

The modification of surfaces with polymers is a passive strategy to render them non-fouling. It is of high interest for a broad range of applications because it is a non-toxic and environmentally friendly method. However non-fouling surfaces have to withstand harsh conditions, therefore the binding chemistry of the polymer to the substrate has to be as strong as possible. Although many surface binding groups, such as thiols[1] and silanes[2], with a sufficient adhesion force are already known, they are often very specific towards certain substrates. Recently new, promising adhesive molecules based on catechols were found in the adhesion part of mussels[3]. They were successfully coupled to poly (ethylene glycol) and adsorbed on metal oxide substrates[4]. It is suggested that depending on the substrate additional functional groups are involved in the adhesion process[5]. This is very interesting in respect to find a broadly applicable adhesive.

In order to enhance the search for such novel adhesive groups a new screening protocol was developed within this project. It consists of a new device for parallel testing in aqueous solutions, the creation of a potential adhesive library and an efficient semi quantitative read-out based on fluorescence.

Eighty parallel incubation experiments can be performed using the actual device. Potential adhesive groups are coupled to poly(L-lysine)-grafted-poly(ethylene glycol) (PLL- $g$-PEG), this allows a broad combination of various potential adhesives. The functional read out is done by a test with a fluorescently labeled protein whereas the intensity gives a semi-quantitative conclusion about the adhesion properties.

[1] S.D. Evans, A. Ulman, Chem.Phys.Lett. 1990, 170, 462

[2] L.H.Lee, J.Colloid Interface Sci. 1968, 27, 751

[3] J.H.Waite, Integr.Comp.Biol.. 2002, 42, 1172.

[4] J.L.Dalsin,P.B.Messersmith, Mater.Today. 2005, 38

[5] V.Vreelan, J.H.Waite, L.Epstein, J.Phycol. 1998, 34, 1 